IJMMS 31:11 (2002) 675-686

PII. S0161171202107022

http://ijmms.hindawi.com

(c) Hindawi Publishing Corp.

\title{
MULTIPLE QUANTUM PRODUCTS IN TORIC VARIETIES
}

\section{HOLGER SPIELBERG}

Received 11 July 2001 and in revised form 26 December 2001

We generalize the author's formula for Gromov-Witten invariants of symplectic toric manifolds (1999) to those needed to compute the quantum product of more than two classes directly, that is, involving the pullback of the Poincaré dual of the point class in the DeligneMumford spaces $\bar{M}_{0, m}$.

2000 Mathematics Subject Classification: 53D45, 14N35.

1. Introduction. Let $(X, \omega)$ be a symplectic manifold with compatible almostcomplex structure $J$. If $g$ and $m$ are nonnegative integers, we denote by $\bar{M}_{g, m}$ the Deligne-Mumford space of genus- $g$ curves with $m$ marked points. If, furthermore, $A \in H_{2}(X, \mathbb{Z})$ denotes a degree-2 homology class of $X, \mu_{g, m}(X, A)$ will be the space of stable genus- $g J$-holomorphic maps to $X$ with homology class $A$. The Gromov-Witten invariants of $X$ (cf. [2, 9]) are multilinear maps

$$
\Phi_{g, m}^{X, A}: H^{*}\left(\overline{\mathcal{M}}_{g, m}, \mathbb{Q}\right) \otimes H^{*}(X, \mathbb{Q})^{\otimes m} \longrightarrow \mathbb{Q}
$$

that are defined as follows. Let $\pi: \mathcal{M}_{g, m}(X, A) \rightarrow \bar{M}_{g, m}$ be the natural projection map forgetting the map to $X$ (and stabilizing the curve if necessary). Furthermore, let $\mathrm{ev}_{i}$ : $\mu_{g, m}(X, A) \rightarrow X$ be the evaluation map at the $i$ th marked point, that is, the map that sends a stable map $\left(C ; x_{1}, \ldots, x_{m} ; f\right)$ to $f\left(x_{i}\right) \in X$. Then for classes $\beta \in H^{*}\left(\bar{M}_{g, m}, \mathbb{Q}\right)$ and $\alpha_{i} \in H^{*}(X, \mathbb{Q})$, the Gromov-Witten invariants are defined by

$$
\Phi_{g, m}^{X, A}\left(\beta ; \alpha_{1}, \ldots, \alpha_{m}\right)=\int_{\left[\mathcal{M}_{g, m}(X, A)\right]^{\mathrm{virt}}} \pi^{*}(\beta) \wedge \mathrm{ev}_{1}^{*}\left(\alpha_{1}\right) \wedge \cdots \wedge \mathrm{ev}_{m}^{*}\left(\alpha_{m}\right) .
$$

Here the integration on the right-hand side is not over the entire moduli space but over the so-called virtual fundamental class.

For the case of $(X, J)$ being a smooth projective variety with a $\left(\mathbb{C}^{*}\right)$-action, Graber and Pandharipande [5] have proven that Bott-style localization techniques apply to the integral in (1.2). Their techniques can easily be extended to torus actions, so in particular they apply to smooth projective toric varieties (for toric varieties see, e.g., $[4,8])$.

In [11] (also see [12]), using these localization techniques for the virtual fundamental class, we have proven an explicit combinatorial formula of the genus-0 GromovWitten invariants for smooth projective toric varieties for the cases when $\beta=1 \in$ $H^{0}\left(\bar{M}_{0, m}, \mathbb{Q}\right)$. In this paper, we derive a similar formula for the case where the class $\beta$ is the maximal product of (the Chern class of) cotangent lines to the marked points, that is for those classes $\beta$ which are Poincaré dual to a finite number of points in $\bar{M}_{0, m}$. 
Knowing the Gromov-Witten invariants for $\beta=$ P.D.(pt) makes computations of products in the (small) quantum cohomology ring easier. For two cohomology classes $\gamma_{1}, \gamma_{2} \in H^{*}(X, \mathbb{Q})$, their quantum product is defined to be

$$
\gamma_{1} \star \gamma_{2}=\sum_{A \in H_{2}(X, \mathbb{Z})} \sum_{i} \Phi_{0,3}^{X, A}\left(\gamma_{1}, \gamma_{2}, \delta_{i}\right) \delta_{i}^{\vee} q^{A},
$$

where the inner sum runs over a basis $\left(\delta_{i}\right)$ of $H^{*}(X, \mathbb{Q})$. Here $\left(\delta_{i}^{\vee}\right)$ denotes the basis of $H^{*}(X, \mathbb{Q})$ dual to $\left(\delta_{i}\right)$. It is easy to show that the product of more than two classes is given by

$$
\gamma_{1} \star \cdots \star \gamma_{r}=\sum_{A \in H_{2}(X, \mathbb{Z})} \sum_{i} \Phi_{0, r+1}^{X, A}\left(\text { P.D. }(\mathrm{pt}) ; \gamma_{1}, \ldots, \gamma_{r}, \delta_{i}\right) \delta_{i}^{\vee} q^{A},
$$

where P.D.(pt) denotes the Poincaré dual of a point in $\bar{M}_{0, r+1}$. By Witten's conjecture (see [14], proven by Kontsevich in [7]), we know that (a multiple of) the class P.D.(pt) in $H^{*}\left(\bar{M}_{0, r+1}\right)$ can be expressed as the product of cotangent line classes, hence the invariants in (1.4) can be computed directly by the formula proposed in this paper.

The techniques used in this paper also yield the invariants for $\beta$ being a different product of Chern classes of such cotangent line bundles. However, it seems to be much more difficult to formalize such a more general approach. For the sake of a (hopefully) better exposition of the key ideas, we leave the more general case to the interested reader.

The structure of the paper is as follows: in Section 2, we recall some results on toric varieties, mostly to fix our notation. In Section 3, we quickly describe the fixed point components of $\mu_{0, m}\left(X_{\Sigma}, A\right)$ with respect to the action induced from $X_{\Sigma}$. In Section 4 , we recall the localization results for toric varieties, to apply them in Section 5 to the case where $\beta=$ P.D.(pt). In Section 6, we finally give the formula for the GromovWitten invariants for symplectic toric manifolds in this case, and in Section 7, we illustrate the formula on the example of $\mathbb{P}_{\mathbb{P}^{1}}\left(\mathcal{O}^{\oplus(r-2)} \oplus \mathscr{O}(1) \oplus \mathcal{O}(1)\right)$; as an interesting byproduct, we derive the quantum cohomology ring of this variety (also using recent results of [3]), which surprisingly coincides with Batyrev's ring stated in [1].

2. Preliminaries of toric varieties. We will quickly recall some facts about toric varieties and mostly introduce our notation-our standard references for this section are $[1,4,8]$.

Let $X_{\Sigma}$ be a smooth projective toric variety of complex dimension $d$, given by the fan $\Sigma$. Choose a class $\omega$ in the Kähler cone of $X_{\Sigma}$, and let $\Delta_{\omega}$ be the corresponding moment polytope. On the variety $X_{\Sigma}$, the $d$-dimensional torus $T_{N}:=\left(\mathbb{C}^{*}\right)^{d}$ acts effectively, and the (irreducible) subvarieties of $X_{\Sigma}$ that are left invariant under this action are in oneto-one correspondence with the facets of the polytope $\Delta_{\omega}$. Moreover, the $T_{N}$-invariant divisors (which are in one-to-one correspondence to the $(d-1)$-dimensional faces of $\left.\Delta_{\omega}\right)$ generate the cohomology ring $H^{*}\left(X_{\Sigma}, \mathbb{Z}\right)$ of $X_{\Sigma}$-we will denote the $(d-1)$ dimensional faces of $\Delta_{\omega}$ by $Z_{1}, \ldots, Z_{n}$. We also remind the reader that the relations between these divisors in the cohomology ring are given by the combinatorics of $\Delta_{\omega}$ or equivalently, by that of the fan $\Sigma$. For (higher-degree) cohomology classes, we will sometimes use multi-index notation, that is, $Z^{l}$ expands to $Z_{1}^{l_{1}} \cdots Z_{n}^{l_{n}}$. 
We will be using the weights of the torus action on the tangent bundle at fixed points of $X_{\Sigma}$. The vertices of $\Delta_{\omega}$ are in one-to-one correspondence with these fixed points, and we will usually denote these vertices by the Greek letter $\sigma$. For any vertex $\sigma$, there are exactly $d$ edges $e_{1}, \ldots, e_{d}$ in $\Delta_{\omega}$ that meet at $\sigma$. Each edge of the polytope $\Delta_{\omega}$ correspondents to $T_{N}$-invariant $\mathbb{C P}^{1}$ in $X_{\Sigma}$. Then the tangent space $T_{\sigma} X_{\Sigma}$ at $\sigma$ splits $T_{N}$-invariantly into the tangent lines along these subvarieties. If we denote by $\sigma_{1}, \ldots, \sigma_{d}$ the vertices that are connected by the edges $e_{1}, \ldots, e_{d}$ to $\sigma$, we will denote by $\omega_{\sigma_{i}}^{\sigma}$ the weight of the $T_{N}$-action on $T_{\sigma} X_{\Sigma}$ into the direction of $e_{i}$.

When referring to a degree-2 homology class $\lambda \in H_{2}\left(X_{\Sigma}, \mathbb{Z}\right)$, we will usually give its intersection vector $\left(\lambda_{i}\right)_{i=1, \ldots, n}$ with the divisor classes $Z_{i}$, that is, $\lambda_{i}:=\left\langle Z_{i}, \lambda\right\rangle$. Note, however, that the $\lambda_{i}$ have to satisfy certain linear relations to represent a degree-2 homology class. In fact, we have that $\operatorname{dim} H_{2}\left(X_{\Sigma}, \mathbb{Z}\right)=\operatorname{dim} H^{2}\left(X_{\Sigma}, \mathbb{Z}\right)=n-d$.

3. Fixed-point components of the induced action on $\mu_{0, m}\left(X_{\Sigma}, A\right)$. Remember that an element of $\mu_{0, m}\left(X_{\Sigma}, A\right)$ is (up to isomorphisms) a tuple $\left(C ; x_{1}, \ldots, x_{m} ; f\right)$ where $C$ is an algebraic curve of genus zero with singularities at most ordinary double points, $x_{i} \in C_{\text {smooth }}$ are marked points, and $f: C \rightarrow X_{\Sigma}$ is the map to the variety $X_{\Sigma}$. The $T_{N^{-}}$ action on $X_{\Sigma}$ then induces an action of $T_{N}$ on $\mathcal{M}_{0, m}\left(X_{\Sigma}, A\right)$ by simple composition with the map $f$, that is, $t \cdot\left(C ; x_{1}, \ldots, x_{m} ; f\right)=\left(C ; x_{1}, \ldots, x_{m} ; t \circ f\right)$, where

$$
t \circ f: C \stackrel{f}{\longrightarrow} X_{\Sigma} \stackrel{\varphi_{t}}{\longrightarrow} X_{\Sigma}
$$

is the composition of $f$ with the diffeomorphism $\varphi_{t}$ given by the action of $t$ on $X_{\Sigma}$.

It is then easy to see (cf. [11]) that the image of a fixed point $(C ; \underline{x} ; f) \in M_{0, m}\left(X_{\Sigma}, A\right)$ must be left invariant by the $T_{N}$-action, or in other words, it has to live on the 1skeleton of $\Delta_{\omega}$. Moreover, the marked points $x_{i}$ of such a stable map will have to be mapped to fixed points $\sigma_{i}$ in $X_{\Sigma}$. The fixed-point components of $\mu_{0, m}\left(X_{\Sigma}, A\right)$ can then be characterized by the so-called $\mu_{0, m}\left(X_{\Sigma}, A\right)$-graphs $\Gamma$ (see [11, Definition 6.4])-these are graphs on the 1-skeleton on $\Delta_{\omega}$, without loops, with decorations representing the position of the marked points and the multiplicities of the map to $X_{\Sigma}$ on the irreducible components. If $\Gamma$ is such a graph, we will usually denote by $M_{\Gamma}$ the product of DeligneMumford spaces corresponding to the graph $\Gamma$, which is up to a finite automorphism group $\mathrm{A}_{\Gamma}$ isomorphic to the fixed-point component in $\mu_{0, m}\left(X_{\Sigma}, A\right)$ corresponding to $\Gamma$.

We recall the following lemma from [13] describing the automorphism group $A_{\Gamma}$.

LEMmA 3.1. The automorphism group $\mathrm{A}_{\Gamma}$ of the universal curve stack $\pi: \mathscr{C}_{\Gamma} \rightarrow \mu_{\Gamma}$ fits into the following exact sequence of groups:

$$
1 \rightarrow \prod_{e \in \operatorname{Edge}(\Gamma)} \mathbb{Z}_{d_{e}} \rightarrow \mathrm{A}_{\Gamma} \longrightarrow \operatorname{Aut}(\Gamma) \longrightarrow 1
$$

where the group of graph automorphisms Aut $(\Gamma)$ acts naturally on $\prod_{e} \mathbf{Z}_{d(e)}, \mathbf{A}_{\Gamma}$ being the semidirect product. Here $d(e)$ is the multiplicity of the edge e in the graph $\Gamma$, and the action of $\mathbf{Z}_{d(e)}$ on $M_{\Gamma}$ is induced by the usual action of $\mathbf{Z}_{d(e)}$ on a map $\mathbb{P}^{1} \rightarrow \mathbb{P}^{1}$ of orderd $(e)$.

Furthermore, the map

$$
\gamma / \mathbf{A}_{\Gamma}: M_{\Gamma} / \mathbf{A}_{\Gamma} \longrightarrow M_{0, m}\left(X_{\Sigma}, A\right)
$$


induced by the obvious map $\gamma: M_{\Gamma} \rightarrow M_{0, m}\left(X_{\Sigma}, A\right)$ is a closed immersion of DeligneMumford stacks, and its image is a component of the $T_{N}$-fixed point stack of $M_{0, m}\left(X_{\Sigma}, A\right)$.

4. Localization for the genus- $0 \mathrm{GW}$ invariants of toric varieties. In the setup described in the previous section, Graber and Pandharipande's virtual localization formula applies: if $V$ is an equivariant vector bundle on the moduli space $\mu_{0, m}\left(X_{\Sigma}, A\right)$, then

$$
\int_{\left[\mathcal{M}_{0, m}\left(X_{\Sigma}, A\right)\right]^{\mathrm{virt}}} e^{T_{N}}(V)=\sum_{\Gamma} \frac{\iota_{*} e^{T_{N}}\left(\left.V\right|_{\mathcal{M}_{\Gamma}}\right)}{e^{T_{N}}\left(\mathcal{N}_{\Gamma}^{\text {virt }}\right)},
$$

where $\mathcal{N}_{\Gamma}$ is the so-called virtual normal bundle to $\mu_{\Gamma}$ and $e^{T_{N}}$ denotes the equivariant Euler class. Since each $\mathrm{ev}_{i}^{*}\left(Z_{j}\right)$ is equal to the (standard) Euler class of an equivariant line bundle over $\mathcal{M}_{0, m}\left(X_{\Sigma}, A\right)$, we obtain

$$
\Phi_{0, m}^{X_{\Sigma}, A}\left(Z^{l_{1}}, \ldots, Z^{l_{m}}\right)=\sum_{\Gamma} \frac{1}{\left|\mathbf{A}_{\Gamma}\right|} \int_{\mathcal{M}_{\Gamma}} \frac{\prod_{j=1}^{m} \prod_{k=1}^{n}\left(\omega_{k}^{\sigma(j)}\right)^{l_{j, k}}}{e^{T_{N}}\left(\mathcal{N}_{\Gamma}^{\mathrm{virt}}\right)},
$$

where $\omega_{k}^{\sigma(j)}$ is the following weight: suppose that the vertex $\sigma(j)$, the $j$ th marked point mapped to in $\Delta_{\omega}$, is given by $\sigma(j)=Z_{i_{1}} \cdots Z_{i_{d}}$ with $i_{l} \neq i_{l^{\prime}}$ whenever $l \neq l^{\prime}$. If $k \notin\left\{i_{1}, \ldots, i_{d}\right\}$, then $\omega_{k}^{\sigma(j)}:=0$. Otherwise suppose that (without loss of generality) $i_{d}=k$ and let $\hat{\sigma}(j)$ be the unique vertex given by $\hat{\sigma}(j)=Z_{i_{1}} \cdots Z_{i_{d-1}} Z_{i_{d+1}}$ such that $i_{d+1} \neq i_{l}$ for all $l$. Then $\omega_{k}^{\sigma(j)}:=\omega_{\hat{\sigma}(j)}^{\sigma(j)}$.

By a careful analysis of the virtual normal bundle (see [11, Theorem 7.2]), we can compute its equivariant Euler class-before we will give its formula here, we fix some notation. For an edge $e \in \operatorname{Edge}(\Gamma)$, define

$$
\Lambda_{\Gamma}(e):=\frac{(-1)^{h} h^{2 h}}{(h !)^{2}\left(\omega_{\sigma_{d}}^{\sigma}\right)^{2 h}} \prod_{j=1}^{d-1} \frac{\prod_{k=\lambda_{i_{j}+1}}^{-1}\left(\omega_{\sigma_{j}}^{\sigma}-(k / h) \cdot \omega_{\sigma_{d}}^{\sigma}\right)}{\prod_{k=0}^{\lambda_{i_{j}}}\left(\omega_{\sigma_{j}}^{\sigma}-(k / h) \cdot \omega_{\sigma_{d}}^{\sigma}\right)} .
$$

In this formula, we use the following notation: the edge $e$ connects the two fixed points $\sigma$ and $\sigma_{d}$ with multiplicity $h$. The indices $i_{j}$ and $\hat{i}_{j}$ are chosen pairwise different such that $\sigma=Z_{i_{1}} \cdots Z_{i_{d}}$ and $\sigma_{j}=Z_{i_{1}} \cdots Z_{\hat{i}_{j}} \cdots Z_{i_{d}}\left(Z_{i_{j}}\right.$ is replaced by $\left.Z_{\hat{i}_{j}}\right)$. The homology class of the edge $e$ is given by $\lambda=\left(\lambda_{1}, \ldots, \lambda_{n}\right)$, in particular $\lambda_{i_{j}}=e \cdot Z_{i_{j}}$.

Furthermore, let $\operatorname{Vert}_{t, s}(\Gamma)$ be the set of vertices $v$ in the graph $\Gamma$ with $t$ edges and $s$ marked points. As usual, we will call the pair $(v, e)$ of a vertex $v$ and an edge $e$ at the vertex $v$ a flag and denote it by $F=(v, e)$. For a vertex $v \in \operatorname{Vert}_{t, s}(\Gamma)$, we will denote the $t$ flags containing $v$ by $F_{1}(v), \ldots, F_{t}(v)$, and if $t=1$ we will usually omit the index. Moreover, for any flag $F=(v, e)$, we can naturally associate a weight $\omega_{F}$ : if the other vertex of the edge $e$ is $v^{\prime}$, and if the images by the moment map of vertices $v$ and $v^{\prime}$ are the vertices $\sigma, \sigma^{\prime}$ of $\Delta_{\omega}$, then $\omega_{F}:=\omega_{\sigma^{\prime}}^{\sigma}$. Finally, let $\operatorname{val}(v):=t, \operatorname{deg}(v):=s+t$, and define the class

$$
\omega(v):= \begin{cases}\omega_{F(v)} & \text { if } t=1, s=0, \\ 1 & \text { if } t=s=1, \\ \left(\omega_{F_{1}(v)}+\omega_{F_{2}(v)}\right)^{-1} & \text { if } t=2, s=0, \\ \left(\left(\omega_{F_{1}(v)}-e_{F_{1}(v)}\right) \cdots\left(\omega_{F_{t}(v)}-e_{F_{t}(v)}\right)\right)^{-1} & \text { if } t+s \geq 3 .\end{cases}
$$


Here the $e_{F}$ are Euler classes of universal cotangent lines to marked points of $\mu_{\Gamma}=$ $\prod_{v \in \operatorname{Vert}(\Gamma)} \bar{M}_{0, \operatorname{deg}(v)}$, and the indices (from 1 through $t$, for $\operatorname{val}(v)=t$ ) refer to the different edges leaving the vertex $v$.

For notational convenience, we also define the weight polynomials $\omega_{\text {total }}^{\sigma(v)}$ and $\omega_{\text {total }}^{F(v)}$ as follows (see [13]). Let $\sigma(v)=Z_{i_{1}} \cdots Z_{i_{d}}$ and $\sigma_{j}=Z_{i_{1}} \cdots Z_{\hat{i}_{j}} \cdots Z_{i_{d}}$ as above. Then the two weight polynomials are defined to be

$$
\begin{aligned}
& \omega_{\text {total }}^{\sigma(v)}:=\prod_{j=1}^{d} \omega_{\sigma_{j}}^{\sigma}, \\
& \omega_{\text {total }}^{F(v)}:=\frac{1}{\omega_{F_{1}(v)}}+\cdots+\frac{1}{\omega_{F_{\mathrm{val}(v)}(v)}} .
\end{aligned}
$$

Note that $\omega_{\text {total }}^{\sigma(v)}$ only depends on the vertex $\sigma(v) \in \Delta_{\omega}$, the vertex of $\Delta_{\omega}$ where $v$ is mapped to.

Proposition 4.1 (see [11]). With this notation, the inverse of the equivariant Euler class of the virtual normal bundle has the following expression:

$$
e^{T_{N}}\left(\mathcal{N}_{\Gamma}\right)^{-1}=\left[\prod_{t, s} \prod_{v \in \operatorname{Vert}_{t, s}(\Gamma)}\left(\omega_{\text {total }}^{\sigma(v)}\right)^{t-1} \cdot \omega(v)\right] \cdot \prod_{e \in \operatorname{Edge}(\Gamma)} \Lambda_{\Gamma}(e) .
$$

Proposition 4.2 (see $[12,13])$. The integral over $\mu_{\Gamma}$ of the inverse of the equivariant Euler class of the virtual normal bundle equals

$$
\int_{\mathcal{M}_{\Gamma}} e^{T_{N}}\left(\mathcal{N}_{\Gamma}\right)^{-1}=\left[\prod_{t, s} \prod_{v \in \operatorname{Vert}_{t, s}(\Gamma)} \frac{\left(\omega_{\text {total }}^{\sigma(v)}\right)^{t-1} \cdot\left(\omega_{\text {total }}^{F(v)}\right)^{t+s-3}}{\prod_{i=1}^{t} \omega_{F_{i}(v)}}\right] \prod_{e \in \operatorname{Edge}(\Gamma)} \Lambda_{\Gamma}(e) .
$$

In particular, we see that if we want to generalize formula (4.2) to nontrivial classes $\beta \in H^{*}\left(\bar{M}_{0, m}, \mathbb{Q}\right)$, the localization formula (4.1) tells us that it suffices to compute the equivariant Euler classes of the restrictions of the equivariant bundles on $\mu_{0, m}\left(X_{\Sigma}, A\right)$ representing the class $\beta$, combine this class with the equivariant Euler class of the virtual normal bundle, and integrate over $\mu_{\Gamma}$.

5. Cotangent line bundles and their restrictions to the fixed point components. In this section, we study how pullbacks of certain classes $\beta \in H^{*}\left(\bar{M}_{0, m}\right)$ localize to fixed-point components $M_{\Gamma}$.

Let $\overline{\mathscr{C}}_{0, m} \rightarrow \bar{M}_{0, m}$ be the universal curve, and let $x_{i}: \bar{M}_{0, m} \rightarrow \overline{\mathscr{C}}_{0, m}$ be the marked point sections $(i=1, \ldots, m)$. We will denote by $\mathbb{L}_{i} \rightarrow \bar{M}_{0, m}$ the $i$ th universal cotangent line, that is, the pullback by $x_{i}$ of the relative cotangent bundle $K_{\overline{\mathscr{C}}_{0, m} / \overline{\mathbb{M}}_{0, m}}$ :

$$
\mathbb{L}_{i}:=x_{i}^{*}\left(K_{\bar{\complement}_{0, m} / \bar{M}_{0, m}}\right) \text {. }
$$


For simplicity, we restrict ourselves here to maximal sums of the line bundles $\mathbb{a}_{i}$, that is, to those of which the rank is equal to $\operatorname{dim} \bar{M}_{0, m}=m-3$. By Kontsevich's theorem [7], we know that in this case

$$
\int_{\bar{M}_{0, m}} e\left(\mathbb{L}_{1}^{\oplus d_{1}} \oplus \cdots \oplus \mathbb{L}_{m}^{\oplus d_{m}}\right)=\frac{(m-3) !}{d_{1} ! \cdots d_{m} !},
$$

that is, the Euler class of this bundle is Poincaré dual to $(m-3) ! /\left(d_{1} ! \cdots d_{m} !\right)$ points; it is exactly this kind of classes $\beta$ we need in order to compute quantum products of more than two factors (see (1.4)). Note that the $d_{i}$ 's fulfill the equation $d_{1}+\cdots+d_{m}=$ $m-3$.

LEMmA 5.1. The map $\pi: \mu_{0, m}\left(X_{\Sigma}, A\right) \rightarrow \bar{M}_{0, m}$ forgetting the map to $X_{\Sigma}$ is equivariant with respect to the induced $T_{N}$-action on $M_{0, m}\left(X_{\Sigma}, A\right)$ and the trivial action on $\bar{M}_{0, m}$.

Proof. Since the $T_{N}$-action on $\mu_{0, m}\left(X_{\Sigma}, A\right)$ is induced from the action on the image of the curve in $X_{\Sigma}$ (and which is discarded by the map $\pi$ ), this is obvious.

COROLLARY 5.2. The pullback by $\pi$ of any bundle $E$ on $\bar{M}_{0, m}$ is an equivariant bundle on $\mu_{0, m}\left(X_{\Sigma}, A\right)$ with trivial fiber action.

REMARK 5.3. Corollary 5.2 implies, in particular, that the equivariant and the nonequivariant Euler classes of such pullback bundles coincide; we will therefore use them interchangeably in these cases.

LEMMA 5.4. Let $E \rightarrow \bar{M}_{0, m}$ be a vector bundle of a vector bundle of rank $\mathrm{rk} E=m-3$. If $\pi\left(M_{\Gamma}\right) \neq \bar{M}_{0, m}$, then $e\left(\left.\pi^{*} E\right|_{\mu_{\Gamma}}\right)=0$.

Proof. If $\pi\left(\mu_{\Gamma}\right) \neq \bar{M}_{0, m}$, then the codimension of $\pi\left(\mu_{\Gamma}\right) \subset \bar{M}_{0, m}$ is at least one. Therefore

$$
e\left(\left.\pi^{*} E\right|_{\mu_{\Gamma}}\right)=\pi^{*} e\left(\left.E\right|_{\pi\left(\mu_{\Gamma}\right)}\right)=\pi^{*}(0)=0,
$$

since $\operatorname{rk} E>\operatorname{dim} \pi\left(\mathcal{M}_{\Gamma}\right)$.

The lemma implies that if $e\left(\left.\pi^{*} E\right|_{\mathcal{M}_{\Gamma}}\right) \neq 0$ for a bundle $E$ with $\operatorname{rk} E=m-3$, the graph $\Gamma$ contains only one vertex $v_{\Gamma}$ that corresponds to a stable component under the projection $\pi$ to $\bar{M}_{0, m}$. In other words, if we fix $v_{\Gamma}$ as a root of the graph $\Gamma$, all its branches contain at most one marked point. We will call such graphs $\Gamma$ simple.

THEOREM 5.5. Let $\Gamma$ be a simple $\mu_{0, m}\left(X_{\Sigma}, A\right)$-graph, and let $v_{\Gamma}$ be the unique stable vertex of $\Gamma$ and $\tilde{m}=\operatorname{deg}\left(v_{\Gamma}\right)$ its degree. The indices of the marked points $\tilde{x}_{1}, \ldots, \tilde{x}_{\tilde{m}}$ of $\bar{M}_{0, \tilde{m}}$ are chosen such that $\tilde{x}_{1}, \ldots, \tilde{x}_{m}$ are mapped by $\pi$ to the marked points $x_{1}, \ldots, x_{m}$ in $\bar{M}_{0, m}$, respectively. Furthermore, let $\mathscr{L}_{i} \rightarrow \bar{M}_{0, \tilde{m}}$ be the cotangent lines to the marked points of $\bar{M}_{0, \tilde{m}}$. Then

$$
\begin{aligned}
e\left(\left.\pi^{*}\left(\mathbb{L}_{1}^{\oplus d_{1}} \oplus \cdots \oplus \mathbb{L}_{m}^{\oplus d_{m}}\right)\right|_{\mu_{\Gamma}}\right) e\left(\mathscr{L}_{m+1}^{\oplus d_{m+1}} \oplus \cdots \oplus \mathscr{L}_{\tilde{m}}^{\oplus d_{\tilde{m}}}\right) \\
=e\left(\mathscr{L}_{1}^{\oplus d_{1}} \oplus \cdots \oplus \mathscr{L}_{m}^{\oplus d_{m}} \oplus \cdots \oplus \mathscr{L}_{\tilde{m}}^{\oplus d_{\tilde{m}}}\right)
\end{aligned}
$$

whenever $d_{1}+\cdots+d_{m}=m-3$ and $d_{1}+\cdots+d_{\tilde{m}}=\tilde{m}-3$. 
PROoF. First of all notice that $\pi$ factors through the projection map $\mu_{\Gamma} \rightarrow \bar{M}_{0, \tilde{m}}$ to the factor corresponding to the vertex $v_{\Gamma}$. Hence it suffices to consider the behaviour of cotangent line bundles under $\pi_{\tilde{m}, m}: \bar{M}_{0, \tilde{m}} \rightarrow \bar{M}_{0, m}$, forgetting the last $(\tilde{m}-m)$ points. This map $\pi_{\tilde{m}, m}$ again factors into

$$
\pi_{\tilde{m}, m}: \bar{M}_{0, \tilde{m}} \stackrel{\pi_{\tilde{m}}}{\longrightarrow} \bar{M}_{0, \tilde{m}-1} \stackrel{\pi_{\tilde{m}-1}}{\longrightarrow} \cdots \stackrel{\pi_{m+1}}{\longrightarrow} \bar{M}_{0, m} .
$$

We will prove the statement for the map $\pi_{m+1}$-the theorem then follows by induction and Kontsevich's theorem (see (5.2)).

So we want to show that (in the case $d_{1}+\cdots+d_{m}=m-3$ )

$$
e\left(\pi_{m}^{*}\left(\mathscr{L}_{1}^{\oplus d_{1}} \oplus \cdots \oplus \mathscr{L}_{m}^{\oplus d_{m}}\right) \oplus \mathscr{L}_{m+1}\right)=e\left(\mathscr{L}_{1}^{\oplus d_{1}} \oplus \cdots \oplus \mathscr{L}_{m}^{\oplus d_{m}} \oplus \mathscr{L}_{m+1}\right) .
$$

It is well known (see, e.g., [6]) that

$$
e\left(\mathscr{L}_{i}\right)=e\left(\pi_{m+1}^{*}\left(\mathscr{L}_{i}\right)\right)+D_{i}
$$

where $D_{i} \cong \bar{M}_{0,3} \times \bar{M}_{0, m}$ is the divisor in $\bar{M}_{0, m+1}$ where the $\bar{M}_{0,3}$-bubble contains the marked points $x_{i}$ and $x_{m+1}$. Now note that $\left.\mathscr{L}_{m+1}\right|_{D_{i}}$ is constant for any $i=1, \ldots, m$, hence $e\left(\mathscr{L}_{m+1}\right) \cdot D_{i}=0$. This yields (5.6).

6. The formula for the Gromov-Witten invariants. The next corollary summarizes what we have shown so far.

COROLLARY 6.1. Let $E$ be the vector bundle $E=\mathbb{L}_{1}^{\oplus d_{1}} \oplus \cdots \oplus \mathbb{\mathbb { L } _ { m } ^ { \oplus }} d_{m}$ on $\bar{M}_{0, m}$ such that $d_{1}+\cdots+d_{m}=m-3$. Let $\beta=e(E)$ be the Euler class of $E$. Then

$$
\Phi_{0, m}^{X_{\Sigma}, A}\left(\beta ; Z^{l_{1}}, \ldots, Z^{l_{m}}\right)=\sum_{\Gamma \text { simple }} \frac{1}{\left|\mathbf{A}_{\Gamma}\right|} \int_{\mathcal{M}_{\Gamma}} \frac{e\left(\left.\pi^{*} E\right|_{\mu_{\Gamma}}\right) \prod_{j, k=1}^{m, n}\left(\omega_{k}^{\sigma(j)}\right)^{l_{j, k}}}{e^{T_{N}}\left(\mathcal{N}_{\Gamma}^{\text {virt }}\right)} .
$$

We will now compute the integral over the fixed-point components to obtain an explicit formula for these Gromov-Witten invariants.

THEOREM 6.2. Let $\Gamma$ be a simple $\mu_{0, m}\left(X_{\Sigma}, A\right)$-graph, and let $v_{\Gamma}$ be the unique stable vertex of $\Gamma$. Let $r: \operatorname{Vert}(\Gamma) \rightarrow \mathbb{N}$ be the map defined by

$$
r(v):= \begin{cases}m-3 & \text { if } v=v_{\Gamma} \\ 0 & \text { otherwise. }\end{cases}
$$

As before let $d_{i}$ be nonnegative integers such that $d_{1}+\cdots+d_{m}=m-3$. If $E=\mathbb{L}_{1}^{\oplus d_{1}} \oplus$ $\cdots \oplus \mathbb{L}_{m}^{\oplus d_{m}}$, then the following formula holds:

$$
\begin{aligned}
\int_{\mathcal{M}_{\Gamma}} \frac{e^{T_{N}}\left(\left.\pi^{*}(E)\right|_{\mathcal{M}_{\Gamma}}\right)}{e^{T_{N}}\left(\mathcal{N}_{\Gamma}\right)}= & \frac{\left(\operatorname{deg}\left(v_{\Gamma}\right)-3\right) !}{d_{1} ! \cdots d_{m} !\left(\operatorname{deg}\left(v_{\Gamma}\right)-m\right) !} \cdot \prod_{e \in \operatorname{Edge}(\Gamma)} \Lambda_{\Gamma}(e) \\
& \cdot\left[\prod_{t, s} \prod_{v \in \operatorname{Vert}_{t, s}(\Gamma)} \frac{\left(\omega_{\text {total }}^{\sigma(v)}\right)^{t-1} \cdot\left(\omega_{\text {total }}^{F(v)}\right)^{t+s-r(v)-3}}{\prod_{i=1}^{t} \omega_{F_{i}(v)}}\right] .
\end{aligned}
$$


Proof. By Kontsevich's formula (5.2), it suffices to consider $d_{1}=m-3, d_{2}=\cdots=$ $d_{m}=0$. In this case, by Theorem 5.5 , the left-hand side of (6.3) equals

$$
\left[\int_{\mathcal{M}_{\Gamma}} e\left(\mathscr{L}_{1, v_{\Gamma}}^{\oplus m-3}\right) \cdot \prod_{t, s} \prod_{v \in \operatorname{Vert}_{t, s}(\Gamma)}\left(\omega_{\text {total }}^{\sigma(v)}\right)^{t-1} \cdot \omega(v)\right] \cdot \prod_{e \in \operatorname{Edge}(\Gamma)} \Lambda_{\Gamma}(e) .
$$

We therefore have to prove that

$$
\begin{aligned}
\int_{\mathcal{M}_{\Gamma}} e\left(\mathscr{L}_{1, v_{\Gamma}}^{\oplus m-3}\right) \cdot \prod_{v \in \operatorname{Vert}(\Gamma)} \omega(v) \\
=\frac{\left(\operatorname{deg}\left(v_{\Gamma}\right)-3\right) !}{(m-3) !\left(\operatorname{deg}\left(v_{\Gamma}\right)-m\right) !} \prod_{t, s} \prod_{v \in \operatorname{Vert}_{t, s}(\Gamma)} \frac{\left(\omega_{\text {total }}^{F(v)}\right)^{t+s-r(v)-3}}{\prod_{i=1}^{t} \omega_{F_{i}(v)}} .
\end{aligned}
$$

For the case that $v \in \operatorname{Vert}_{t, s}(\Gamma)$ is different from $v_{\Gamma}$, we have shown in the proof of Proposition 4.2 (see [13]) that

$$
\int_{\bar{M}_{0, \operatorname{deg}(v)}} \omega(v)=\frac{\left(\omega_{\text {total }}^{F(v)}\right)^{t+s-3}}{\prod_{i=1}^{t} \omega_{F_{i}(v)}}
$$

Hence we only have to consider the case when $v=v_{\Gamma}$; since it is very similar to the previous case, we will only outline its proof. As in [13], let $P_{n}\left(x_{1}, \ldots, x_{k}\right)=\sum_{\sum_{i} \tilde{d}_{i}=n} \prod_{j} x_{j} \tilde{d}_{j}$. Let $t:=\operatorname{val}\left(v_{\Gamma}\right), \tilde{m}:=\operatorname{deg}\left(v_{\Gamma}\right)$, and $r:=m-3$. We will also write $F_{j}$ instead of $F_{j}\left(v_{\Gamma}\right)$. Note that for the vertex $v_{\Gamma}$, we always have $\tilde{m}-r-3 \geq 0$. Therefore

$$
\begin{aligned}
\int_{\bar{M}_{0, \tilde{m}}} \omega\left(v_{\Gamma}\right) e\left(\mathscr{L}_{1}^{\oplus r}\right) & =\int_{\bar{M}_{0, \tilde{m}}} e\left(\mathscr{L}_{1}^{\oplus r}\right) \prod_{j=1}^{t} \frac{1}{\omega_{F_{j}}-e_{F_{j}}} \\
& =\int_{\bar{M}_{0, \tilde{m}}} e\left(\mathscr{L}_{1}^{\oplus r}\right) \prod_{j=1}^{t} \frac{1}{\omega_{F_{j}}} \sum_{i=0}^{\infty}\left(\frac{e_{F_{j}}}{\omega_{F_{j}}}\right)^{i} \\
& =\prod_{j=1}^{t} \frac{1}{\omega_{F_{j}}} \sum_{\sum \tilde{d}_{i}=\tilde{m}-r-3} \frac{(\tilde{m}-3) !}{\tilde{d}_{1} ! \cdots \tilde{d}_{t} ! r !}\left(\frac{1}{\omega_{F_{1}}}\right)^{\tilde{d}_{1}} \cdots\left(\frac{1}{\omega_{F_{t}}}\right)^{\tilde{d}_{t}} \\
& =\frac{(\tilde{m}-3) !}{r !(\tilde{m}-r-3) !} \cdot \frac{\left(\omega_{\text {total }}^{F\left(v_{\Gamma}\right)}\right)^{\tilde{m}-r-3}}{\prod_{i=1}^{t} \omega_{F_{i}\left(v_{\Gamma}\right)}}
\end{aligned}
$$

which finishes the proof.

7. Example: the quantum cohomology of $\mathbb{P}_{\mathbb{P}^{1}}\left(\mathcal{O}^{\oplus(r-2)} \oplus \mathscr{O}(1) \oplus \mathscr{O}(1)\right)$. In this section, we want to illustrate how useful the extension of the formula to the case where $\beta=$ P.D.(pt) really is for the computation of the quantum cohomology ring of a toric manifold. 
In [10], Siebert and Tian have shown that if the ordinary cohomolgy ring of a symplectic manifold $X$ is given by

$$
H^{*}(X, \mathbb{Q})=\mathbb{Q}\left[Z_{1}, \ldots, Z_{k}\right] /\left\langle R_{1}, \ldots, R_{l}\right\rangle,
$$

where $R_{1}, \ldots, R_{l}$ are relations, then the quantum ring is given by

$$
Q H^{*}(X, \mathbb{Q})=\mathbb{Q}\left[Z_{1}, \ldots, Z_{k}\right] /\left\langle R_{1}^{*}, \ldots, R_{l}^{*}\right\rangle,
$$

where $R_{i}^{*}$ are the relations $R_{i}$, but evaluated with respect to the quantum product instead of the cup product.

Consider the toric manifolds of the form $\mathbb{P}_{\mathbb{P} 1}\left(\mathcal{O}^{\oplus(r-2)} \oplus \mathcal{O}(1) \oplus \mathcal{O}(1)\right)$. In [3], Costa and Miró-Roig have studied the three-point Gromov-Witten invariants of these manifolds and announced that they will derive the quantum cohomology ring of these manifolds in an upcoming paper. We have chosen the same example to illustrate how the formula derived in this note can make computations much easier.

We will recall some properties of $\mathbb{P}_{\mathbb{P} 1}\left(\mathcal{O}^{\oplus(r-2)} \oplus \mathcal{O}(1) \oplus \mathcal{O}(1)\right)$, where $r$ is an integer greater than or equal to three (for more details see [3]). Its cohomology ring is given by

$$
H^{*}\left(X_{\Sigma}, \mathbb{Q}\right)=\mathbb{Q}\left[Z_{1}, \ldots, Z_{r+2}\right] /\left\langle L_{1}, L_{3}, \ldots, L_{r+1}, P_{1}, P_{2}\right\rangle,
$$

where the relations are given by $L_{1}=Z_{1}-Z_{2}, L_{3}=Z_{3}-Z_{r+2}, \ldots, L_{r-1}=Z_{r-1}-Z_{r+2}$, $L_{r}=Z_{2}+Z_{r}-Z_{r+2}, L_{r+1}=Z_{2}+Z_{r+1}-Z_{r+2}, P_{1}=Z_{1} Z_{2}, P_{2}=Z_{3} \cdots Z_{r+2}$.

To see this, consider the fan whose one-dimensional cones are (with respect to some basis $e_{1}, \ldots, e_{r}$ in the lattice $\left.\mathbb{Z}^{r}\right) v_{1}=e_{1}, v_{2}=-e_{1}+e_{r-1}+e_{r}, v_{3}=e_{2}, \ldots, v_{r+1}=e_{r}$ and $v_{r+2}=-e_{2}-\cdots-e_{r}$, and whose set of primitive collections is given by

$$
\mathfrak{P}=\left\{\left\{v_{1}, v_{2}\right\},\left\{v_{3}, \ldots, v_{r+2}\right\}\right\} .
$$

In [3, Proposition 3.6], Costa and Miró-Roig obtain (in what follows, we will freely use their notation)

$$
Z_{1} \star Z_{1}=\left(Z_{r+2} \star Z_{r+2}-2 Z_{1} \star Z_{r+2}\right) \sum_{i \geq 1} q_{1}^{i}
$$

Hence, we will only have to compute the quantum product $Z_{3} \star \cdots \star Z_{r+2}$ to get a presentation of the quantum cohomology ring. To do so, we will have to compute the Gromov-Witten invariants of the form

$$
\Phi_{0, r+1}^{a \lambda_{1}+b \lambda_{2}}\left(\text { P.D.(pt); } Z_{3}, \ldots, Z_{r+2}, \gamma\right) .
$$

In formula (7.6), the two homology classes $\lambda_{1}$ and $\lambda_{2}$ are given by the intersection vectors

$$
\lambda_{1}=(1,1,0, \ldots, 0,-1,-1,0), \quad \lambda_{2}=(0,0,1, \ldots, 1) .
$$

LEMMA 7.1. If $b \neq 1$, then all invariants of the form (7.6) are zero. 
Proof. Suppose that for given $a, b$, and $\gamma$, the invariant (7.6) is nonzero. Since $\left\langle c_{1}\left(X_{\Sigma}\right), a \lambda_{1}+b \lambda_{2}\right\rangle=2 r b$, we must have $2 r+\operatorname{deg} \gamma=2 r b+2 r$. However, the real dimension of $X_{\Sigma}$ is equal to $2 r$, hence $0 \leq \operatorname{deg} \gamma \leq 2 r$, and therefore $b \leq 1$.

Now suppose $b$ is zero and therefore $\operatorname{deg} \gamma=0$ as well, that is $\gamma$ is a multiple of the trivial class $1 \in H^{0}\left(X_{\Sigma}\right)$. If $a=0$ as well, then the invariant is zero since it just equals the cup product of the cohomology classes $Z_{3}, \ldots, Z_{r+2}$. So suppose $a>1$. Then

$$
\begin{aligned}
\Phi_{0, r+1}^{a \lambda_{1}} & \text { (P.D. (pt); } \left.Z_{3}, \ldots, Z_{r+2}, 1\right) \\
= & \Phi_{0, r}^{a \lambda_{1}}\left(\text { P.D. (pt); } Z_{3}, \ldots, Z_{r-1}, Z_{r+2}, Z_{r}, Z_{r+1}\right) \\
= & \sum_{\substack{a_{1}+\cdots+a_{r-2}=a \\
j_{1}, \ldots, j_{r-3}}} \Phi_{0,3}^{a_{1} \lambda_{1}}\left(Z_{3}, Z_{r+1}, \gamma_{j_{1}}\right) \Phi_{0,3}^{a_{2} \lambda_{1}}\left(Z_{4}, \gamma_{j_{1}}^{\vee}, \gamma_{j_{2}}\right) \cdots \Phi_{0,3}^{a_{r-2}}\left(Z_{r+2}, Z_{r}, \gamma_{j_{r-3}}^{\vee}\right),
\end{aligned}
$$

where the $\gamma_{j_{i}}$ run over a basis of $H^{*}\left(X_{\Sigma}, \mathbb{Z}\right)$. Since $a>0$, at least one of $a_{i}$ has to be positive. On the other hand, we have

$$
\left\langle Z_{3}, \lambda_{1}\right\rangle=\cdots=\left\langle Z_{r-1}, \lambda_{1}\right\rangle=\left\langle Z_{r+2}, \lambda_{1}\right\rangle=0
$$

So, as soon as $a_{i}>0$, the corresponding three-point invariant in the sum above is zero. This proves the lemma.

LEMмA 7.2. If $a>0$, then all invariants of the form (7.6) are zero.

Proof. Suppose that for given $a, b$ and $\gamma$, the invariant (7.6) is nonzero. Then, by the previous lemma, $b=1$. Hence we have to consider homology classes $a \lambda_{1}+\lambda_{2}$, and $\gamma$ being a multiple of the class of top degree, say $Z_{1} Z_{3} \cdots Z_{r} Z_{r+2}$.

The vertices of the moment polytope of $X_{\Sigma}$ are $\sigma_{i, j}:=Z_{i} \cdot Z_{3} \cdots \widehat{Z_{j}} \cdots Z_{r+2}$ where $i \in\{1,2\}$ and $j \in\{3, \ldots, r+2\}$. As usual, the hat on top of $Z_{j}$ signals its omission. Hence, the 1-skeleton of the moment polytope of $X_{\Sigma}$ has the following edges:

(1) for $3 \leq t<s \leq r+2$, edges between $\sigma_{1, t}$ and $\sigma_{1, s}$, and between $\sigma_{2, t}$ and $\sigma_{2, s}$, all having homology class $\lambda_{2}$;

(2) for $3 \leq t \leq r-1$ or $t=r+2$, an edge between $\sigma_{1, t}$ and $\sigma_{2, t}$ of homology class $\lambda_{1}$

(3) for $t=r, r+1$, an edge between $\sigma_{1, t}$ and $\sigma_{2, t}$ of homology class $\lambda_{1}+\lambda_{2}$.

The readers will now easily convince themselves that there is no simple graph $\Gamma$ in this class such that $Z_{3}, \ldots, Z_{r-1}, Z_{r+1}, Z_{r+1}, Z_{r+2}$ and $Z_{1} Z_{3} \cdots Z_{r}, Z_{r+2}$ all have nonzero equivariant Euler class on $M_{\Gamma}$, unless $a=0$. Finally note that $Z_{r}=Z_{r+1}$ as cohomology classes, which finishes the proof.

LEMMA 7.3. The following equation holds: $\Phi_{0, r+1}^{\lambda_{2}}$ (P.D.(pt); $Z_{3}, \ldots, Z_{r-1}, Z_{r+1}, Z_{r+1}$, $\left.Z_{r+2}, Z_{1} Z_{3} \cdots Z_{r} Z_{r+2}\right)=1$.

Proof. The only simple graph $\Gamma$ such that $Z_{3}, \ldots, Z_{r-1}, Z_{r+1}, Z_{r+1}, Z_{r+2}$ and $Z_{1} Z_{3} \cdots Z_{r} Z_{r+2}$ all have nonzero equivariant Euler class on $\Gamma$ is the one with one edge between $\sigma_{1, r+1}$ and $\sigma_{1, r}$ where all but the last marked point are at $\sigma_{1, r}$. Applying the formula derived in this note yields the following for the Gromov-Witten invariant 


$$
\begin{aligned}
\Phi:= & \left.\Phi_{0, r+1}^{\lambda_{2}} \text { (P.D.(pt); } Z_{3}, \ldots, Z_{r-1}, Z_{r+1}, Z_{r+1}, Z_{r+2}, Z_{1} Z_{3} \cdots Z_{r} Z_{r+2}\right): \\
\Phi= & -\frac{\omega_{\sigma_{2, r+1}}^{\sigma_{1, r+1}} \omega_{\sigma_{1,3}}^{\sigma_{1, r+1}} \cdots \omega_{\sigma_{1, r}}^{\sigma_{1, r+1}} \omega_{\sigma_{1, r+2}}^{\sigma_{1, r+1}} \omega_{\sigma_{1,3}}^{\sigma_{1, r}} \cdots \omega_{\sigma_{1, r} 1}^{\sigma_{1, r}}\left(\omega_{\sigma_{1, r+1}}^{\sigma_{1, r}}\right)^{2} \omega_{\sigma_{1, r+2}}^{\sigma_{1, r}}}{\omega_{\sigma_{1, r+1}}^{\sigma_{1, r}}\left(\omega_{\sigma_{1, r+1}}^{\sigma_{1, r}}\right)^{2} \omega_{\sigma_{1, r+2}}^{\sigma_{1, r}}\left(\omega_{\sigma_{1, r+2}}^{\sigma_{1, r}}-\omega_{\sigma_{1, r}}^{\sigma_{1, r}}\right) \omega_{\sigma_{1, r+2}}^{\sigma_{1, r+1}}} \\
& \cdot \frac{1}{\omega_{\sigma_{1,3}}^{\sigma_{1, r}}\left(\omega_{\sigma_{1,3}}^{\sigma_{1, r}}-\omega_{\sigma_{1, r+1}}^{\sigma_{1, r}}\right) \cdots \omega_{\sigma_{1, r-1}}^{\sigma_{1, r}}\left(\omega_{\sigma_{1, r-1}}^{\sigma_{1, r}}-\omega_{\sigma_{1, r+1}}^{\sigma_{1, r}}\right)} \\
= & \frac{\omega_{\sigma_{1,3}}^{\sigma_{1, r+1}} \cdots \omega_{\sigma_{1, r-1}}^{\sigma_{1, r+1}} \omega_{\sigma_{1, r+2}}^{\sigma_{1, r+1}}}{\left(\omega_{\sigma_{1,3}}^{\sigma_{1, r}}-\omega_{\sigma_{1, r+1}}^{\sigma_{1, r}}\right) \cdots\left(\omega_{\sigma_{1, r-1}}^{\sigma_{1, r}}-\omega_{\sigma_{1, r+1}}^{\sigma_{1, r}}\right)\left(\omega_{\sigma_{1, r+2}}^{\sigma_{1, r}}-\omega_{\sigma_{1, r+1}}^{\sigma_{1, r}}\right)} \\
= & 1,
\end{aligned}
$$

where we have used $\omega_{\sigma_{1, i}}^{\sigma_{1, r+1}}=\omega_{\sigma_{1, i}}^{\sigma_{1, r}}-\omega_{\sigma_{1, r+1}}^{\sigma_{1, r}}$ for $i=3, \ldots, r-1, r+2$ by a similar argument as in [3, Lemma 2.1], that is by applying [11, Lemma 6.7].

COROLLARY 7.4. The quantum cohomology ring of $X_{\Sigma}=\mathbb{P}_{\mathbb{P 1}}\left(\mathcal{O}^{\oplus(r-2)} \oplus \mathscr{O}(1) \oplus \mathbb{O}(1)\right)$ (for $r \geq 3$ ) is given by

$$
Q H^{*}\left(X_{\Sigma}, \mathbb{Z}\right)=\mathbb{Z}\left[Z_{1}, \ldots, Z_{r+2}, q_{1}, q_{2}\right] /\left\langle L_{1}, L_{3}, \ldots, L_{r-1}, R_{1}^{*}, R_{2}^{*}\right\rangle,
$$

where the relations $R_{1}^{\star}$ and $R_{2}^{\star}$ are given by

$$
\begin{aligned}
& R_{1}^{\star}=Z_{1} \star Z_{2}-Z_{r} \star Z_{r+1} q_{1}, \\
& R_{2}^{\star}=Z_{3} \star \cdots \star Z_{r+2}-q_{2},
\end{aligned}
$$

that is, this quantum ring coincides with Batyrev's ring in [1].

Proof. The relation $Z_{3} \star \cdots \star Z_{r+2}=q_{2}$ follows directly from the previous three lemmas. Now consider the other multiplicative relation

$$
\begin{aligned}
Z_{1} \star Z_{2} & =Z_{1} \star Z_{1} \quad \text { since } Z_{1}=Z_{2} \\
& =\sum_{i \geq 1}\left(Z_{r+2} \star Z_{r+2}-2 Z_{1} \star Z_{r+2}\right) q_{1}^{i} \quad \text { by }(7.5) \\
& =\left(Z_{r} \star Z_{r+1}-Z_{1} \star Z_{2}\right) \sum_{i \geq 1} q_{1}^{i} \quad \text { by the linear relations. }
\end{aligned}
$$

Hence we obtain

$$
Z_{1} \star Z_{2} \sum_{i \geq 0} q_{1}^{i}=Z_{r} \star Z_{r+1} \sum_{i \geq 1} q_{1}^{i}
$$

and by comparing the coefficients of the $q_{1}^{i}$, the relation follows.

REMARK 7.5. Note that although we derive the same presentation for the quantum cohomology ring as the one stated by Batyrev in [1], the Gromov-Witten invariants that enter as structure constants into the computation of the quantum products are different from those numbers considered by Batyrev. 
ACKNOWLEDGments. The author is grateful to Laura Costa and Rosa M. Miró-Roig for having sent and explained their paper [3] to him. He also wants to thank the Centro de Analíse Matemática, Geometria e Sistemas Dinâmicos at Instituto Superior Técnico, Lisboa, for its hospitality.

\section{REFERENCES}

[1] V. V. Batyrev, Quantum cohomology rings of toric manifolds, Astérisque 218 (1993), 934.

[2] K. Behrend, Gromov-Witten invariants in algebraic geometry, Invent. Math. 127 (1997), no. 3, 601-617.

[3] L. Costa and R. Miró-Roig, GW-invariants and quantum products with infinitely many quantum corrections, to appear in Forum Math.

[4] W. Fulton, Introduction to Toric Varieties, Annals of Mathematics Studies, vol. 131, Princeton University Press, New Jersey, 1993.

[5] T. Graber and R. Pandharipande, Localization of virtual classes, Invent. Math. 135 (1999), no. 2, 487-518.

[6] J. Harris and I. Morrison, Moduli of Curves, Graduate Texts in Mathematics, vol. 187, Springer-Verlag, New York, 1998.

[7] M. Kontsevich, Intersection theory on the moduli space of curves and the matrix Airy function, Comm. Math. Phys. 147 (1992), no. 1, 1-23.

[8] T. Oda, Convex Bodies and Algebraic Geometry, Ergebnisse der Mathematik und ihrer Grenzgebiete 3 Folge, vol. 15, Springer-Verlag, Berlin, 1988.

[9] Y. Ruan and G. Tian, A mathematical theory of quantum cohomology, J. Differential Geom. 42 (1995), no. 2, 259-367.

[10] B. Siebert and G. Tian, On quantum cohomology rings of Fano manifolds and a formula of Vafa and Intriligator, Asian J. Math. 1 (1997), no. 4, 679-695.

[11] H. Spielberg, A formula for the Gromov-Witten invariants of toric varieties, Ph.D. thesis, Université Louis Pasteur, Département de Mathématique, Institut de Recherche Mathématique Avancée, Strasbourg, 1999.

[12] _ The Gromov-Witten invariants of symplectic toric manifolds, and their quantum cohomology ring, C. R. Acad. Sci. Paris Sér. I Math. 329 (1999), no. 8, 699-704.

[13] _ The Gromov-Witten invariants of symplectic toric manifolds, preprint, 2000 http://arxiv.org/abs/math.AG/0006156.

[14] E. Witten, Two-dimensional gravity and intersection theory on moduli space, Surveys in Differential Geometry (Cambridge, Mass, 1990), vol. 1, Lehigh University, Pennsylvania, 1991, pp. 243-310.

Holger Spielberg: Centro de Analíse Matemática, Geometria e Sistemas DinÂmicos, Instituto Superior TÉCNico, Av. Rovisco PAis, 1049-001 LisboA, PORTUGAL

Current address: KPMG AG, GANGHOFERSTRASSE 29, 80339 MÜNCHEN, GERMANY

E-mail address: Spi e1berg@member . ams . org 


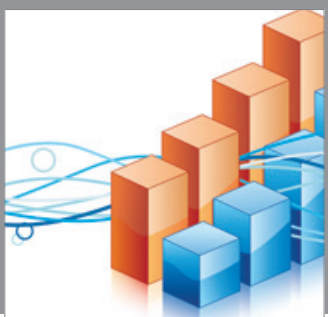

Advances in

Operations Research

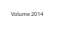

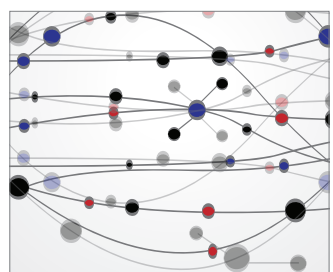

\section{The Scientific} World Journal
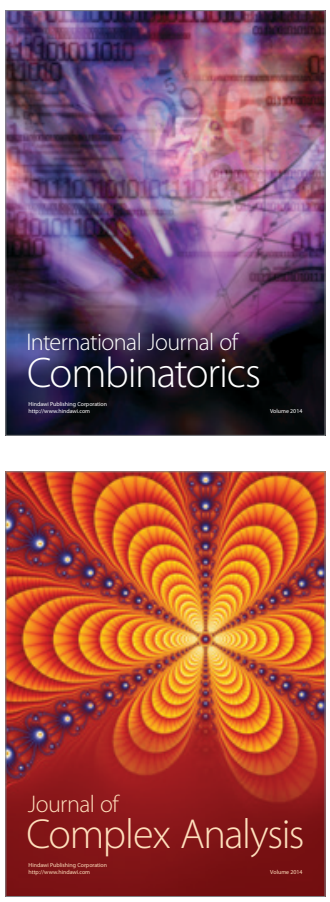

International Journal of

Mathematics and

Mathematical

Sciences
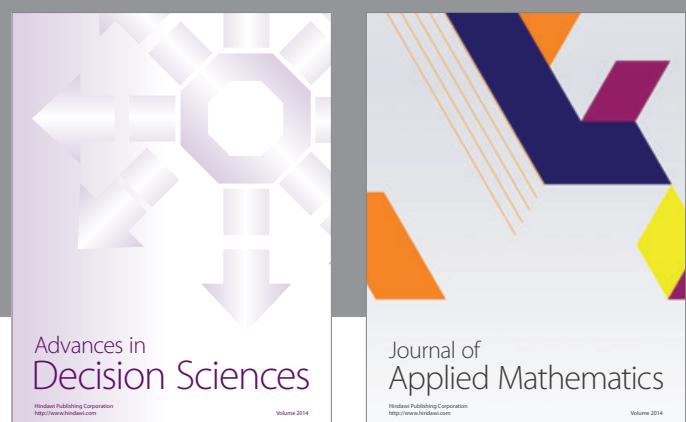

Journal of

Applied Mathematics
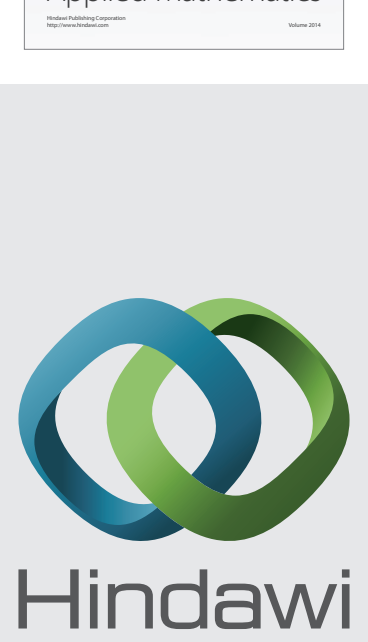

Submit your manuscripts at http://www.hindawi.com
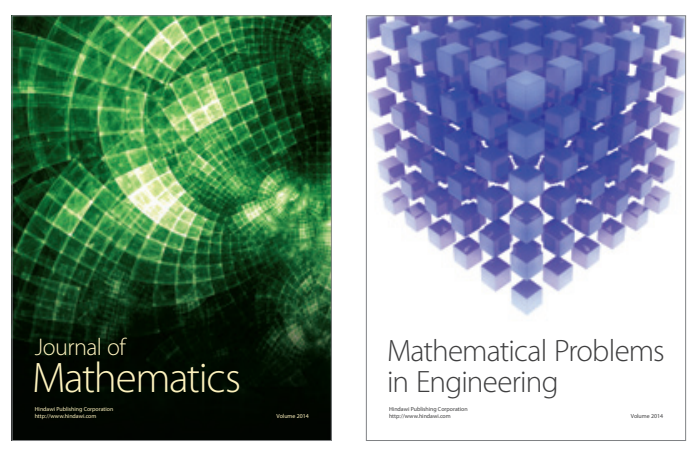

Mathematical Problems in Engineering
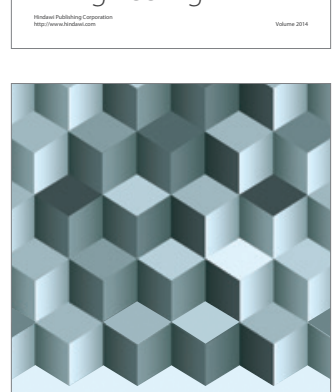

Journal of

Function Spaces
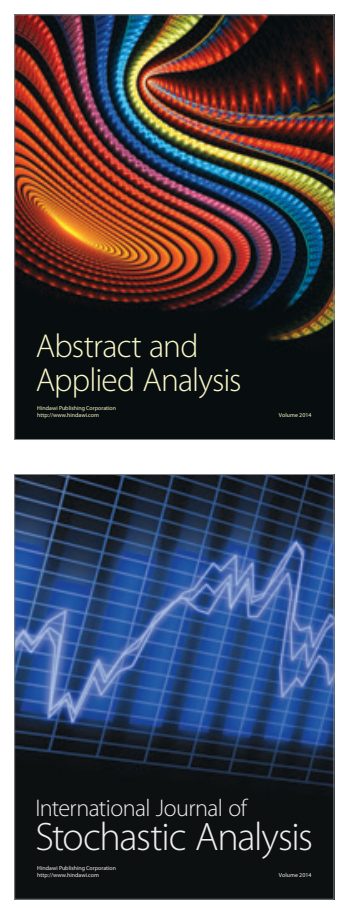

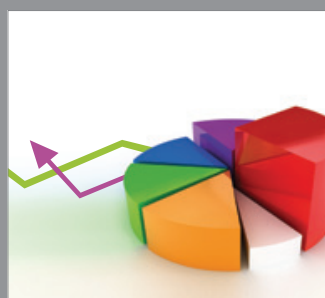

ournal of

Probability and Statistics

Promensencen
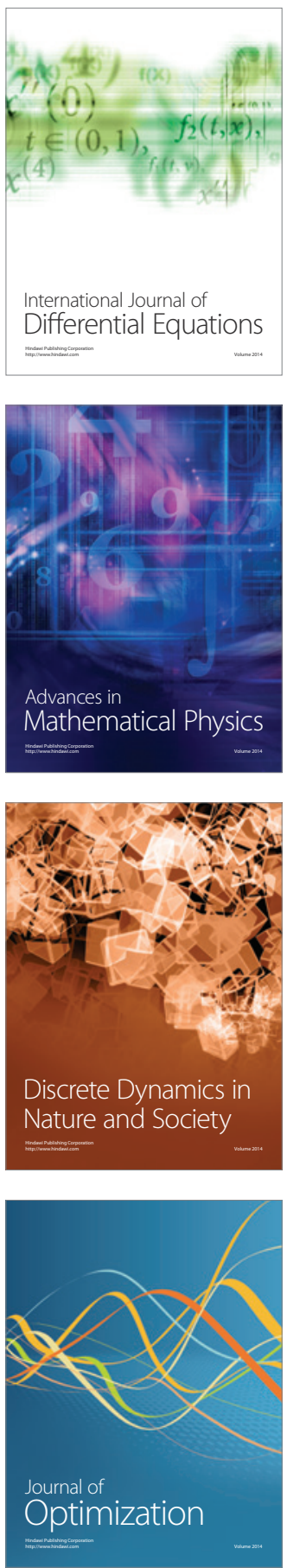